\title{
Resting Energy Expenditure, Insulin Resistance and UCP1 Expression in Human Subcutaneous and Visceral Adipose Tissue of Patients With Obesity
}

\author{
Silvia Bettini ${ }^{1,2 * t}$, Francesca Favaretto ${ }^{1,2 t}$, Chiara Compagnin ${ }^{1,2}$, Anna Belligoli ${ }^{1,2}$, \\ Marta Sanna ${ }^{1,2}$, Roberto Fabris ${ }^{1,2}$, Roberto Serra ${ }^{1,2}$, Chiara Dal Prà ${ }^{1,2}$, Luca Prevedello ${ }^{2}$, \\ Mirto Foletto ${ }^{2}$, Roberto Vettor ${ }^{1,2}$, Gabriella Milan ${ }^{1,2}$ and Luca Busetto ${ }^{1,2}$ \\ ${ }^{1}$ Internal Medicine 3, Department of Medicine, DIMED, University of Padua, Padua, Italy, ${ }^{2}$ Center for the Study and the \\ Integrated Treatment of Obesity, University Hospital of Padua, Padua, Italy
}

\section{OPEN ACCESS}

Edited by:

Massimiliano Caprio,

Università Telematica San

Raffaele, Italy

Reviewed by:

Angelo Cignarelli,

University of Bari Aldo Moro, Italy

Lei Zhang,

Garvan Institute of Medical

Research, Australia

*Correspondence:

Silvia Bettini

d.ssa.silvia.bettini@gmail.com

†These authors have contributed equally to this work

Specialty section:

This article was submitted to

Obesity,

a section of the journal

Frontiers in Endocrinology

Received: 30 April 2019

Accepted: 23 July 2019

Published: 07 August 2019

Citation:

Bettini S, Favaretto F, Compagnin C Belligoli A, Sanna M, Fabris $R$, Serra R, Dal Prà C, Prevedello L,

Foletto $M$, Vettor $R$, Milan $G$ and

Busetto L (2019) Resting Energy

Expenditure, Insulin Resistance and

UCP1 Expression in Human

Subcutaneous and Visceral Adipose

Tissue of Patients With Obesity.

Front. Endocrinol. 10:548.

doi: 10.3389/fendo.2019.00548
Determinants of resting energy expenditure (REE) in humans are still under investigation, especially the association with insulin resistance. Brown adipose tissue (AT) regulates energy expenditure through the activity of the uncoupling protein 1 (UCP1). White AT browning is the process by which some adipocytes within AT depots acquire properties of brown adipocytes ("brite" adipocytes) and it correlates with metabolic improvement. We analyzed determinants of REE in patients with obesity and assessed UCP1 expression as a "brite" marker in abdominal subcutaneous AT (SAT) and visceral omental AT (VAT). Clinical data, REE, free fat mass (FFM), and fat mass (FM) were determined in 209 patients with obesity. UCP1, PPARG coactivator 1 alpha (PPARGC1A), transcription factor A, mitochondrial (TFAM), T-box transcription factor 1 (TBX1), and solute carrier family 27 member 1 (SLC27A1) expression was assayed in SAT and VAT samples, obtained during sleeve gastrectomy from 62 patients with obesity. REE and body composition data were also available for a subgroup of 35 of whom. In 209 patients with obesity a multiple regression model was computed with REE as the dependent variable and sex, waist, FFM, FM, homeostasis model assessment-insulin resistance (HOMA), interleukin-6 and High Density Lipoprotein-cholesterol as the independent variables. Only FFM, FM and HOMA were independently correlated with REE ( $r=0.787$, AdjRsqr $=0.602)$. In each patient VAT displayed a higher UCP1, PPARGC1A, TFAM, TBX1, and SLC27A1 expression than SAT and UCP1 expression in VAT (UCP1-VAT) correlated with Body Mass Index (BMI) $(r=0.287, p<0.05)$. Introducing UCP1-VAT in the multivariate model, we showed that FFM, HOMA, interleukin-6, High Density Lipoprotein-cholesterol, and UCP1-VAT were independent factors correlated with REE ( $r=0.736$, AdjRsqr $=0.612$ ). We confirmed that REE correlates with FFM, FM and HOMA in a large cohort of patients. Our results clearly showed that UCP1-VAT expression was significantly increased in severe human obesity $\left(\mathrm{BMl}>50 \mathrm{~kg} / \mathrm{m}^{2}\right)$ and that it behaved as an independent predictor of REE. Lastly, we suggest that an increased REE and browning in metabolically complicated severe obesity could represent an effort to counteract further weight gain.

Keywords: resting energy expenditure, obesity, insulin resistance, UCP1, browning, adipose tissue 


\section{INTRODUCTION}

Determinants of resting energy expenditure (REE) in humans are still under investigation, especially in patients with severe obesity. Fat free mass (FFM) explains more than $80 \%$ of the interindividual variance in REE (1), but other factors could play a role, such as heritability, hypertension and insulin resistance (2). While an association between REE and insulin resistance (IR) has been previously shown (3-5), in particular in Pima Indians (6), the underlying mechanisms remain unclear.

Brown adipose tissue (BAT) regulates energy expenditure by the dissipation of energy as heat through the activity of the uncoupling protein 1 (UCP1) (7). Several studies have suggested that BAT activation is associated with reduction in blood glucose levels, improvement of IR and increased REE both in animals and in humans (8-10). On the contrary, within white adipose tissue (WAT) depots, visceral adipose tissue (VAT) is a more pathogenic depot compared to subcutaneous adipose tissue (SAT) and increased VAT correlates with a high risk of metabolic syndrome and type 2 diabetes mellitus (T2DM) (11).

Browning is the process by which some adipocytes within WAT depots acquire properties of brown adipocytes (called "beige" or "brite" adipocytes) and studies in mice show a correlation between browning and metabolic improvement (12). While the expression of browning genes in mice is greater in SAT compared to VAT, an opposite pattern of browning gene expression with VAT having higher expression than SAT was observed in humans $(13,14)$. Indeed, UCP1 expression in VAT and SAT (UCP1-VAT and UCP1-SAT), particularly in patients with severe obesity, are still controversial. In the present study, we analyze different populations of patients with severe obesity (PWO) (Figure 1), with the aim of identifying new determinants of REE including UCP1 expression in abdominal SAT and VAT.

\section{RESULTS}

\section{Analyses of REE in 209 Patients With Obesity}

Clinical and laboratory evaluations of the 209 PWO are reported in Table 1. Most of the patients were affected by severe obesity (30 $\mathrm{kg} / \mathrm{m}^{2} \leq$ Body Mass Index (BMI) $<34.99 \mathrm{~kg} / \mathrm{m}^{2}: n=7,3.3 \% ; 35$ $\mathrm{kg} / \mathrm{m}^{2} \leq \mathrm{BMI}<39.99 \mathrm{~kg} / \mathrm{m}^{2}: n=47,22.5 \% ; \mathrm{BMI} \geq 40 \mathrm{~kg} / \mathrm{m}^{2}$ : $n=155,74.2 \%)$.

\footnotetext{
Abbreviations: AT, adipose tissue; BAT, brown adipose tissue; BIA, Body Impedance Assessment; BMI, Body Mass Index; CNHDL, non-HDL-cholesterol; F, female; FFM, Free Fat Mass; FM, Fat mass; FPG, Fasting plasma glucose; HDL, High Density Lipoprotein -cholesterol; HMBS, hydroxymethylbilane synthase; HOMA, Homeostasis model assessment-insulin resistance index; hsCRP, highsensitivity C-Reactive Protein; IL-6, interleukin-6; IR, insulin resistance; LDL, Low Density Lipoprotein -cholesterol; M, male; OGTT, oral glucose tolerance test; PPARGC1A, PPARG coactivator 1 alpha; PWO, patients with obesity; REE, resting energy expenditure; RQ, respiratory quotient; SAT, subcutaneous adipose tissue; SLC27A1, solute carrier family 27 member 1; T2DM, Type 2 diabetes mellitus; TBX1, T-box transcription factor 1; TC, total cholesterol; TFAM, transcription factor A, mitochondrial; TG, triglycerides; TNF- $\alpha$, Tumor Necrosis Factor- $\alpha$; UCP1, uncoupling protein 1; VAT, visceral adipose tissue; VO2, Oxygen Consumption; WAT, white adipose tissue; WC, waist circumference.
}

Self-reported spontaneous physical activity levels were similar among patients.

We divided our population according to their glycaemic profile [based on American Diabetes Association (ADA) (15)] in 3 groups sex and BMI matched: 100 PWO were normoglycaemic, 60 had prediabetes (impaired fasting glycaemia and/or impaired glucose tolerance at the oral glucose tolerance test, OGTT), 49 had T2DM. In the 3 groups REE levels did not display any significant difference even if we found a trend of increase from patients with normoglycaemia $[\mathrm{REE}=1,826(1,618-$ $2,207) \mathrm{kcal} /$ day] to patients with prediabetes [REE $=1,897$ $(1,498-2,314) \mathrm{kcal} / \mathrm{day}]$ and T2DM $[\mathrm{REE}=2,015(1,707-2,340)$ $\mathrm{kcal} /$ day], $p=0.404$.

The REE values were significantly correlated with: $\operatorname{sex}(M=1$ and $F=2 ; r=-0.591, p<0.001)$, weight $(r=0.731$, $p<0.001)$, BMI $(r=0.5, p<0.001)$, waist circumference (WC) $(r=0.616, p<0.001)$, FFM $(r=0.73, p<0.001)$, fat mass $(\mathrm{FM})(r=0.294, p<0.001)$, fasting plasma glucose (FPG) $(r=0.203, p<0.01)$, fasting insulin $(r=0.218$, $p<0.01$ ), homeostasis model assessment-insulin resistance (HOMA) $(r=0.239, p<0.001)$, high-sensitivity C-reactive protein (hs-CRP) $(r=0.228, p<0.01)$, interleukin-6 (IL-6) $(r=0.201, p<0.01)$, and High Density Lipoprotein-cholesterol (HDL-cholesterol) $(r=-0.242, p<0.001)$. To investigate the role of different parameters as predictors of REE, a multiple regression model was computed with REE as the dependent variable and sex, WC, FFM, FM, HOMA, IL-6, and HDLcholesterol as the independent variables. We excluded some interrelated variables: BMI vs. FFM and FM and we selected IL6 values to include inflammatory markers and HOMA data as cumulative valuable index of IR. In this model, FFM, FM and HOMA were the only variables independently correlated with $\operatorname{REE}(r=0.785$, AdjRsqr $=0.601)($ Table 2$)$.

\section{Gene Expression Analysis in Adipose Tissue Depots of 62 Patients With Obesity}

We quantified mRNA expression of genes related to mitochondria and brown/beige adipocytes in paired SAT and VAT of 62 PWO collected during bariatric surgery procedures. Clinical and laboratory evaluations of this group were reported in Table 1.

These patients compared to those described in the previous paragraph displayed a higher BMI range [BMI in 62 patients: 45.4 (42.1-53.4) vs. BMI in 209 patients: 43.9 (39.9-49.4), p $<0.01$ ], including some patients with very high BMI values, and consequently a higher level of circulating leptin. We assessed that WAT of PWO in basal conditions expressed very low levels of UCP1 mRNA $\left(10^{4} / 10^{5}\right.$ times lower) when compared to human VAT surrounding pheochromocytoma that we used as a positive control. In fact, pheochromocytoma is an adrenal neoplasm secreting high levels of catecholamines which strongly stimulate browning and UCP1 expression in the adipose tissue (16). However, we quantified a higher UCP1 expression in VAT compared to SAT of the same patient (Figure 2A) and in several SAT biopsies UCP1 expression resulted undetectable (39/62). In agreement with high UCP1 expression, VAT expressed also 


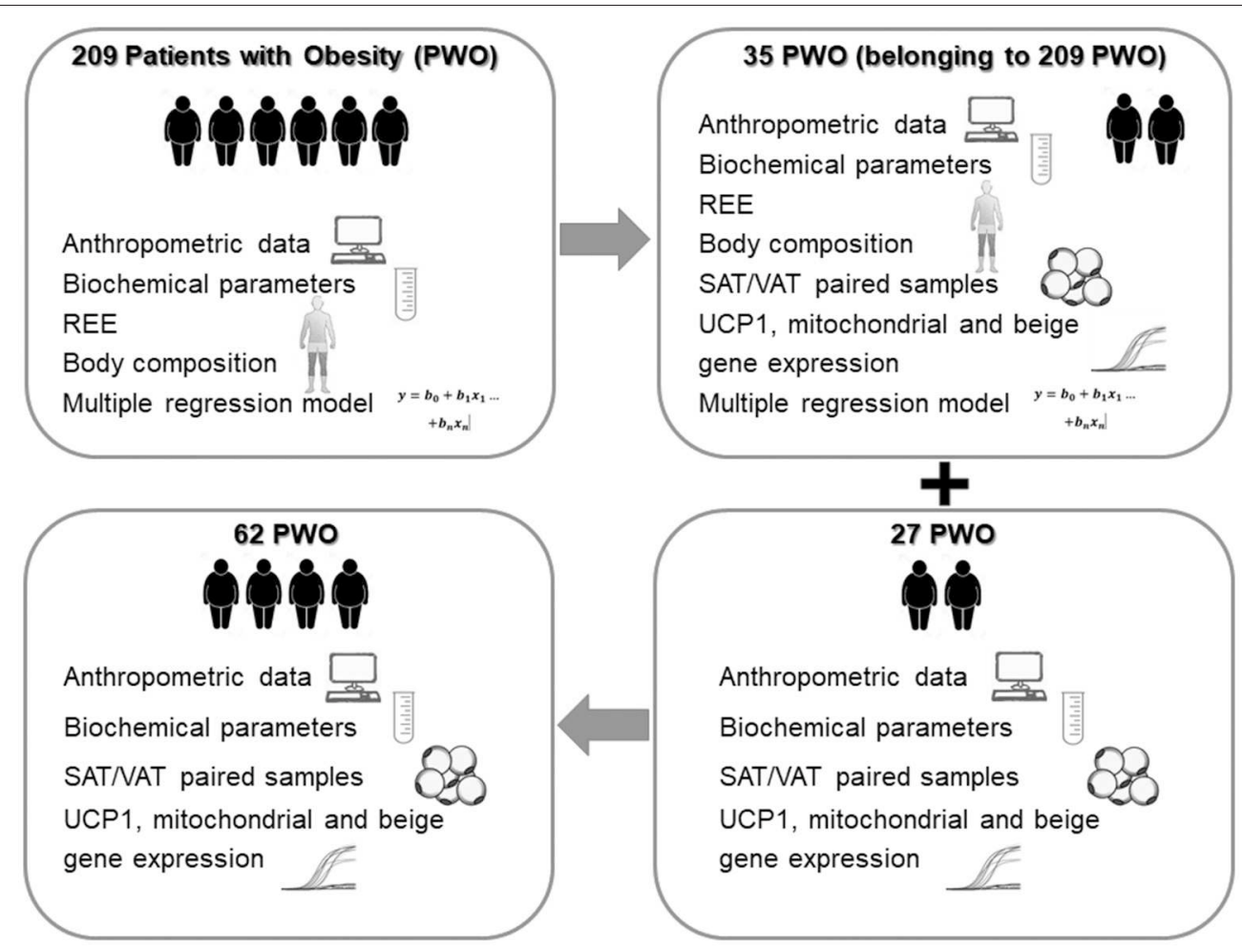

FIGURE 1 | Study design. PWO, Patients with Obesity; REE, resting energy expenditure; SAT, subcutaneous Adipose Tissue; VAT, visceral omental Adipose Tissue; UCP1, uncoupling protein 1 .

higher levels of PPARGC1A and TFAM than SAT (Figures 2B,C). Visceral tissue displayed also a significant increase of the beige-related markers TBX1 and SLC27A1 (Figures 2D,E) when compared to paired SAT.

UCP1-VAT correlated with BMI $(r=0.287, p<0.05)$, as shown in Figure 3.

No further simple correlations were found between UCP1VAT or UCP1-SAT and other clinical and laboratory parameters reported in Table 1. It is worth noting that we highlighted a strong correlations between VAT expression of UCP1 and PPARGC1A $(r=0.465, p<0.001)$, TFAM $(r=0.455, p<0.001)$, TBX1 $(r=0.426, p<0.001)$, and SLC27A1 $(r=0.374, p<0.01)$.

On the basis on the distribution of the UCP1-VAT showed in Figure 3, we divided the PWO into two groups using the BMI cut-off of $50 \mathrm{~kg} / \mathrm{m}^{2}$. In this way, we highlighted that PWO with BMI higher than $50(n=18)$ were characterized by a significantly higher expression of UCP1 in VAT depot compared to PWO with BMI lower than $50(n=43)$ in basal conditions $(p=0.007)$ (Figure 4).

\section{UCP1 Expression in Visceral Omental Adipose Tissue As a Predictor of REE}

In order to study any correlation between UCP1-VAT and REE, we analyzed a subgroup of PWO $(n=35)$, belonging to the main population of 209 PWO where both UCP1 expression and indirect calorimetry for REE quantification were available (Figure 1). The clinical and laboratory parameters of these patients were described in Table 1 and compared with patients of the main population. The statistical analysis clearly showed that the anthropometric characteristics, the biochemical parameters, the body composition and the REE of this subgroup of 35 PWO did not statistically differ from the main population of $209 \mathrm{PWO}$ ( $\mathrm{p}$ column of Table $\mathbf{1}$ ).

In this subgroup of 35 PWO, we confirmed the correlations between REE and weight $(r=0.497, p<0.01)$, BMI $(r=0.366$, $p<0.05)$, WC $(r=0.406, p<0.05)$, FFM $(r=0.45, p<0.01)$ and HOMA $(r=0.472, p<0.01)$. Thus, we applied to this subgroup the same multiple regression model computed for the main population of 209 patients introducing in the analysis the new UCP1-VAT variable as a possible biological implicating factor. PPARGC1A, TFAM, TBX1 and SLC27A1 expression values in VAT were excluded from this model because of their multicollinearity each other and with UCP-VAT. In this new analysis FFM, HOMA, IL-6, HDL-cholesterol and UCP1VAT were the five independent factors correlated with REE $(r=0.736$, AdjRsqr $=0.612)$ (Table 3). Thus, we confirmed the role of FFM and HOMA, previously obtained analyzing the main population of $209 \mathrm{PWO}$, but we also highlighted a possible independent contribution of UCP1-VAT as a determinant of REE. In particular, in the multiple regression model, UCP1-VAT alone explains about $10 \%$ of the variation in REE.

\section{DISCUSSION}

Factors influencing REE in humans are still under investigation, particularly for the mechanisms underlying the association with 
TABLE 1 | Anthropometric characteristics, biochemical parameters, gene expression analyses in white adipose tissue depots, body composition and resting energy expenditure (REE) in 209 patients with obesity (PWO).

\begin{tabular}{|c|c|c|c|c|}
\hline & 209 PWO & 62 PWO & 35 PWO & $p$ \\
\hline $\operatorname{Sex}(M / F)$ & $95 / 114$ & $16 / 46$ & $10 / 25$ & ns \\
\hline Age (y) & $45 \pm 12$ & $46 \pm 12$ & $45 \pm 14$ & ns \\
\hline Weight (kg) & $127.7 \pm 24.8$ & $129 \pm 21.6$ & $120.5 \pm 18.5$ & ns \\
\hline $\mathrm{BMI}\left(\mathrm{kg} / \mathrm{m}^{2}\right)$ & 43.9 (39.9-49.4) & $45.4(42.1-53.4)$ & $45.1 \pm 8.3$ & ns \\
\hline WC (cm) & $131 \pm 15$ & $132 \pm 12$ & $128 \pm 12$ & ns \\
\hline FPG (mmol/l) & $5.8 \pm 1.5$ & $5.8(5.1-7)$ & $5.9 \pm 1.5$ & ns \\
\hline Insulin (mU/I) & $19.9 \pm 12.6$ & 19 (13-29) & $21.4 \pm 13$ & ns \\
\hline HOMA & $5.27 \pm 2.9$ & 4.98 (3.26-7.92) & $5.73 \pm 2.3$ & ns \\
\hline TC (mg/dl) & $192 \pm 37$ & $189 \pm 34$ & $192 \pm 34$ & ns \\
\hline HDL (mg/dl) & $46 \pm 12$ & $47 \pm 12$ & $48 \pm 13$ & ns \\
\hline NHDLC (mg/dl) & $145 \pm 38$ & $142 \pm 33$ & $145 \pm 38$ & ns \\
\hline LDL (mg/dl) & $121(99-138)$ & 119 (95-136) & 117 (97-136) & ns \\
\hline TG (mg/dl) & 110 (79-153) & $121(90-167)$ & $126(89-160)$ & ns \\
\hline hsCRP (mg/l) & $5.58(3.15-10.07)$ & $6.6(3.6-10)$ & $7.2(3.5-11)$ & ns \\
\hline TNF-a (ng/l) & $8.2(6.8-10.6)$ & $8.1(6.5-9.5)$ & $8(6.2-9.2)$ & ns \\
\hline IL-6 (ng/l) & $2.2(1.9-3.7)$ & $3(2.1-4.4)$ & $2.9(2-3.9)$ & ns \\
\hline Leptin $(\mu \mathrm{g} / \mathrm{l})$ & $34 \pm 17$ & $39 \pm 16$ & $36.6 \pm 15$ & ns \\
\hline REE (Kcal/day) & $1,964 \pm 467$ & - & $1,785 \pm 300$ & ns \\
\hline$R Q$ & $0.79 \pm 0.12$ & - & $0.72 \pm 0.08$ & ns \\
\hline FM (kg) & $53.4 \pm 14.32$ & - & $54.6 \pm 11.9$ & ns \\
\hline FM (\%) & $42.7 \pm 8$ & - & $45.6 \pm 7$ & ns \\
\hline FFM (kg) & $71.7 \pm 17.1$ & - & $64.6 \pm 12.6$ & ns \\
\hline FFM (\%) & $57.4 \pm 8.6$ & - & $54.4 \pm 7$ & ns \\
\hline UCP1-SAT & - & $0(0-0.001)$ & $0(0-0.001)$ & - \\
\hline UCP1-VAT & - & $0.006(0.004-0.011)$ & $0.008 \pm 0.005$ & - \\
\hline PPARGC1A-SAT & - & $1.56(1.12-2.42)$ & $1.85(1.3-2.49)$ & - \\
\hline PPARGC1A-VAT & - & $4.5(2.6-6.6)$ & $3.9(1.98-6.54)$ & - \\
\hline TFAM-SAT & - & $0.13(0.09-0.22)$ & $0.14(0.1-0.23)$ & - \\
\hline TFAM-VATAT & - & $0.35(0.25-0.52)$ & $0.32(0.2-0.43)$ & - \\
\hline TBX1-SAT & - & $0.1(0.06-0.16)$ & 0.09 (0.06-0.14) & - \\
\hline TBX1-VAT & - & $0.15(0.074-0.29)$ & $0.15(0.07-0.29)$ & - \\
\hline SLC27A1-SAT & - & $0.37(0.27-0.51)$ & $0.3(0.27-0.5)$ & - \\
\hline SLC27A1-VAT & - & $0.5(0.34-0.72)$ & $0.52 \pm 0.29$ & - \\
\hline
\end{tabular}

M, male; F, female; BMI, Body Mass Index; WC, waist circumference; FPG, Fasting plasma glucose; HOMA, Homeostasis model assessment-insulin resistance index; TC, total cholesterol; HDL, High Density Lipoprotein -cholesterol; NHDLC, non-HDL-cholesterol; LDL, Low Density Lipoprotein -cholesterol; TG, triglycerides; hSCRP, high-sensitivity CReactive Protein; TNF- $\alpha$, Tumor Necrosis Factor- $\alpha$; IL-6, interleukin-6; $R Q$, respiratory quotient; FM, Fat mass; FFM, Free Fat Mass; UCP1, uncoupling protein 1; PPARGC1A, PPARG coactivator 1 alpha; TFAM, transcription factor A, mitochondrial; TBX1, T-box transcription factor 1; SLC27A1, solute carrier family 27 member 1; SAT, subcutaneous adipose tissue; VAT, visceral omental adipose tissue. Data are presented as mean values \pm standard deviations when Normality Test (Shapiro-Wilk) and Equal Variance Test (Brown-Forsythe) have been passed or, if not, as median values (25th-75th percentile). Thirty five PWO's data were compared with 209 PWO's data; statistical analysis was performed with the Mann-Whitney test for independent samples in non-normally distributed variables, independent samples t-test in normally distributed variables and Fisher's exact test in categorical variables; results were reported in the p column.

IR. In Pima Indians basal and 24-h energy expenditure (24hEE) adjusted for body composition, spontaneous physical activity, sex and age are higher in individuals with T2DM compared with
TABLE 2 | Correlation and regression analyses of Resting Energy Expenditure (REE) in 209 patients with obesity.

\begin{tabular}{|c|c|c|}
\hline & \multicolumn{2}{|c|}{ REE (kcal/day) } \\
\hline & Simple linear coefficients $(\boldsymbol{r})$ & Multiple regression coefficients $(\beta)$ \\
\hline Sex & $-0.591^{\star \star \star}$ & -0.131 \\
\hline WC & $0.616^{\star \star \star}$ & 0.0773 \\
\hline FFM & $0.73^{\star \star \star}$ & $0.55^{\star \star \star}$ \\
\hline FM & $0.294^{\star \star \star}$ & $0.199^{\star \star}$ \\
\hline HOMA & $0.239^{\star \star \star}$ & $0.120^{\star}$ \\
\hline HDL & $-0.242^{\star \star \star}$ & 0.0598 \\
\hline IL-6 & $0.201^{\star \star}$ & 0.0785 \\
\hline \multicolumn{3}{|c|}{$\begin{array}{l}\text { WC, waist circumference; FFM, Free Fat Mass (kg); FM, Fat mass (kg); HOMA, } \\
\text { Homeostasis model assessment-insulin resistance index; HDL, High Density Lipoprotein } \\
\text {-cholesterol; IL-6, interleukin-6. Simple linear correlations were calculated by Pearson's } \\
\text { correlation. FM, FFM, and HOMA were independently related to REE in a multiple } \\
\text { regression model }(r=0.785 \text {, AdjRsqr }=0.601) \text {. }{ }^{*} p<0.05 ;{ }^{* \star} p<0.01 ;{ }^{* \star *} p<0.001 \text {. }\end{array}$} \\
\hline
\end{tabular}

non-diabetic subjects (6). In the same way, a study including PWO (mean BMI $32 \mathrm{~kg} / \mathrm{m}^{2}$ ) from Sudan proved that REE increased in the presence of T2DM (5). These recent findings are consistent with what was previously reported by Weyer et al. regarding the direct role of insulin in thermogenesis (17).

The increment of REE described in subjects with high IR level results in lower rates of weight gain (18) due to the increase in fat oxidation rate (19) and in gluconeogenesis induced by high free fatty acids concentration in blood (6). Furthermore, Piaggi et al. (19) described a positive association between FPG, as well as a marker of impaired glucose tolerance, and REE. In support of these mechanisms, the improvement of glycaemic control causes a significant reduction in REE (20).

In this context, our study aims to analyze the association of REE with clinical and laboratory parameters in a large population of Caucasian patients with severe obesity and to quantify UCP1 expression as a "brite" marker in abdominal adipose tissue depots to identify possible determinants of energy expenditure.

We firstly considered all variables correlated with REE in 209 PWO and then we performed a multiple regression model, excluding some interrelated variables. Our results confirm that FFM, which reflects the metabolically active tissue, is the major determinant of $\operatorname{REE}(1,21)$. However, FM and HOMA were also found to be independent predictors of REE. In addition, we found that in our PWO, HOMA is associated with REE both in a simple correlation and in a multiple regression analysis. This result has been supported by the further simple correlations between REE and FPG and insulin, that we showed in the same group. These findings confirm the correlation between REE and HOMA highlighted in other studies $(3,22)$ but our study innovatively enrolled PWO. When we divided our patients according glycaemic profile, we did not find a significant difference in REE between these subgroups. The lack of difference could be accounted for a lower REE in some patients with wellcontrolled T2DM (20) and the concomitant presence of several patients classified as prediabetic with high fasting glycaemia incrementing their REE values (19).

In our study, we also observed a simple correlation between REE and WC. In agreement with literature, WC can be 


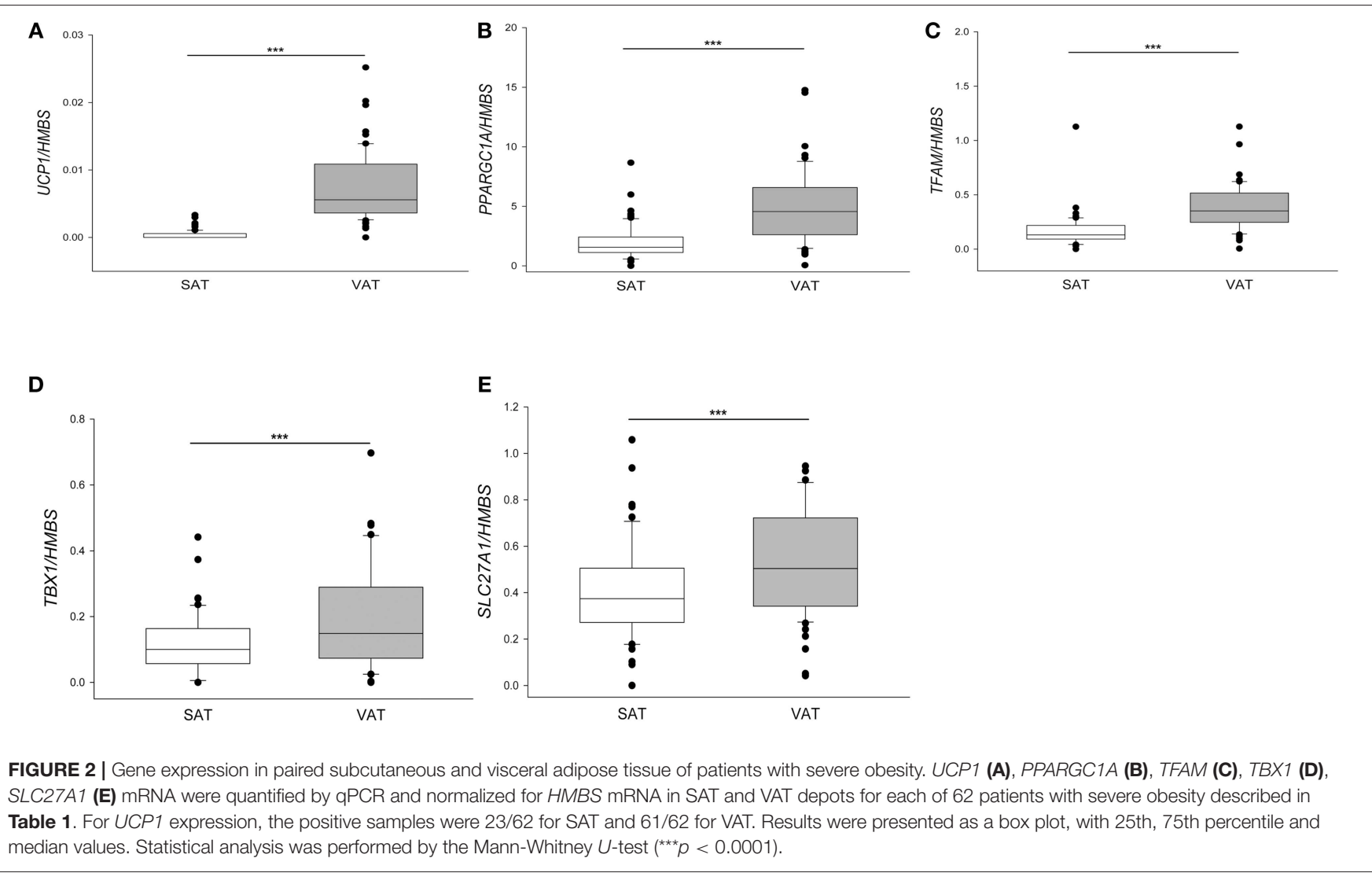

considered as a measure of VAT (23), a fat depot that seems to play a more significant pathogenic role than SAT in the development of metabolic complications. This result is consistent with a previous work, including only women with obesity, showing a relationship between REE and visceral fat accumulation measured by abdominal computed tomography (24). Accordingly, we found a simple inverse correlation between REE and HDL-cholesterol and we recognized HDL as an independent element influencing REE. Our findings underline the strength of the HDL levels, which are properly used in the definition of metabolic syndrome (25), as a clinical marker of IR associated with abdominal adiposity, predictive of cardiovascular diseases and metabolic complications.

It was reported that inflammation was related to REE (26, 27), probably for the energy costs due to the inflammatory status. We considered the blood levels of IL- 6 and hs-CRP as inflammatory markers implicated in obesity and metabolic disorders (28). We found that both factors correlated with REE and we introduced IL-6 levels in our regression model. In fact, new insights supported that IL- 6 not only acts as a central mediator of inflammation but also serves as an endocrine modulator of metabolism for the entire body (29). In mice, IL6 secreted by brown adipocytes was required for effects of BAT on glucose homeostasis (30). Furthermore, very recent findings showed that human beige adipocytes secrete IL- 6 to sustain their own differentiation (31). In PWO increased levels of UCP1 and IL-6 expression in BAT were associated with metabolic improvements (32). Interestingly when we introduce UCP1-VAT in the regression model, IL-6 appears as a further independent variable correlated with REE.

In a group of 62 patients we studied the expression of $U C P 1$, beige-related genes (TBX1 and SLC27A1) and mitochondrial biogenesis markers (PPARGC1A and TFAM), in SAT and VAT depots collected from the same patients during bariatric surgery and thus we explored a possible role of browning in human WAT as a determinant of REE. We found that UCP1 mRNA is higher in VAT than in SAT of unstimulated PWO and that it correlates with BMI, being significantly increased in patients with $\mathrm{BMI}>50 \mathrm{~kg} / \mathrm{m}^{2}$. In our analysis, the positive correlation between BMI and UCP1-VAT could be explained by the high caloric intake of the patients. These results are supported by studies in animal models, where an increase of UCP1 mRNA and protein during the high fat diet was described, mainly in BAT (33). Moreover, a high fat diet increases the expression of UCP1 together with other "brite" markers in WAT of rats (34). Both phenomena could evidence an adaptation that tries to contrast the increase in adiposity. Nevertheless, data regarding browning in both mice and humans are still controversial and, mainly in humans, under investigation. In fact, in humans, browning was observed in SAT of burn patients and during cancer cachexia, two conditions characterized by hypermetabolism $(35,36)$. Furthermore, we know that in lean subjects, with a fluoro-deoxyglucose position emission tomography/computerized tomography analysis, BAT was inversely related to BMI and total and visceral fat areas (37-39). 
We confirmed in PWO that UCP1-VAT was higher than UCP1-SAT as reported previously in humans $(13,14)$. Moreover, we found also an upregulation of beige-related genes (40) (TBX1 and SLC27A1) in VAT, which confirmed that VAT in humans displayed a brite signature in association with increased mitochondrial biogenesis markers (PPARGC1A and TFAM) (41). Jorge et al. (32) demonstrated that PWO expressed higher UCP1 in BAT compared to SAT and they evidenced a simple correlation between UCP1-BAT and oxygen consumption (VO2). Our results seem to indicate that the expression level of UCP1 in VAT could be intermediate between SAT (very low) and BAT (very high) in PWO. Moreover, using a multivariate analysis we were able to show that UCP1-VAT resulted as an independent determinant of REE, explaining about $10 \%$ of the variance in REE. Furthermore, in this model, FM lost its association with REE, suggesting that the contribution of FM to REE could be mediated at least in part by UCP1-VAT. The biological role of UCP1 to predict "brite" proprieties of VAT was corroborated by the strong correlations between UCP1 and PPARGC1A, TFAM, $T B X 1$, and SLC27A1 expression in VAT.

Our findings suggest that, despite mitochondrial degeneration described in IR state (42), UCP1 expression in VAT could contribute to energy expenditure and counteract further weight gain in patients with severe obesity.

Our study has some limitations. In particular, we did not assess other possible causes of variation of REE, like heritability (2), elevated protein metabolism, activated substrate cycle $(4,6,17)$, changes in glucagon levels (43) and central nervous system involvement (44). However, excluding genetic variation, all these proposed explanations can potentially be caused by an IR state $(6,17)$. Secondly, we estimated the FM of PWO but we did not measure the specific amount of VAT and SAT with imaging techniques. Moreover, we considered UCP1 expression in WAT as a well-accepted marker of "brite/beige" adipocytes even though recently other mechanisms driving UCP1-indipendent thermogenesis have been described in WAT of mice and humans (45-49) and it would be interesting to evaluate whether they can also contribute to REE.

In conclusion, we confirmed that REE correlates with FFM, FM and HOMA in a large cohort of patients with severe obesity. Furthermore, we evidenced new independent associations between REE and HDL-cholesterol, IL-6, and UCP1VAT. Moreover, we showed that VAT of patients with obesity expresses more UCP1 than SAT, incrementing with increase of BMI $\left(B M I>50 \mathrm{~kg} / \mathrm{m}^{2}\right)$. Thus, we could hypothesize that abdominal omental VAT in humans is more prone to dietinduced browning. Lastly, we suggest that an increased REE and browning in metabolically complicated severe obesity could represent an effort to counteract further weight gain. Further studies in a larger population analyzing UCP-1 expression upon stimulation could be necessary to confirm and extend our preliminary results.

\section{MATERIALS AND METHODS}

\section{Patients}

The study design was schematically represented in Figure 1.

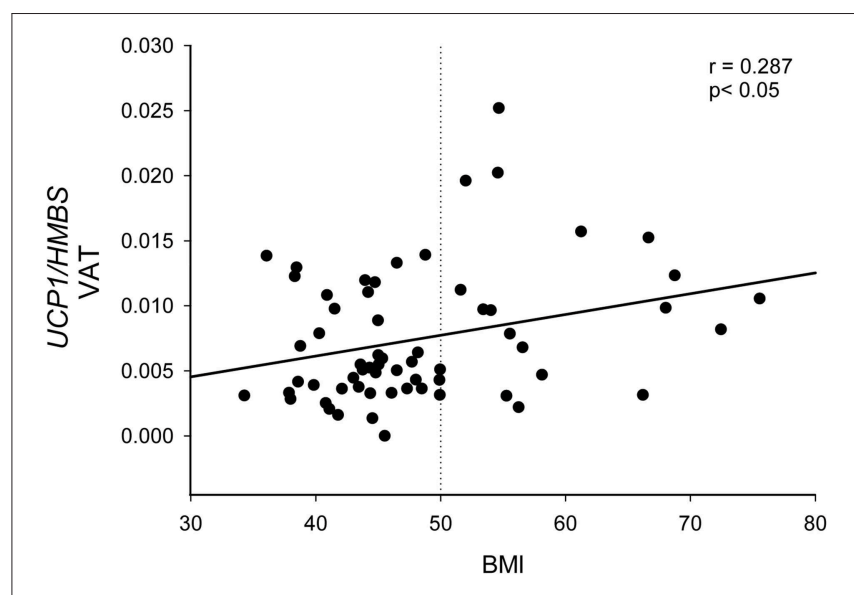

FIGURE 3 | Correlation between UCP1 expression in visceral omental adipose tissue and BMI. UCP1 mRNA expression quantified by qPCR and normalized to HMBS mRNA was correlated with BMI in 61 patients with severe obesity. Statistical analysis was performed by Pearson correlation. The dotted line represents the BMl cut-off of $50 \mathrm{~kg} / \mathrm{m}^{2}$ used to divide patients in Figure 4.

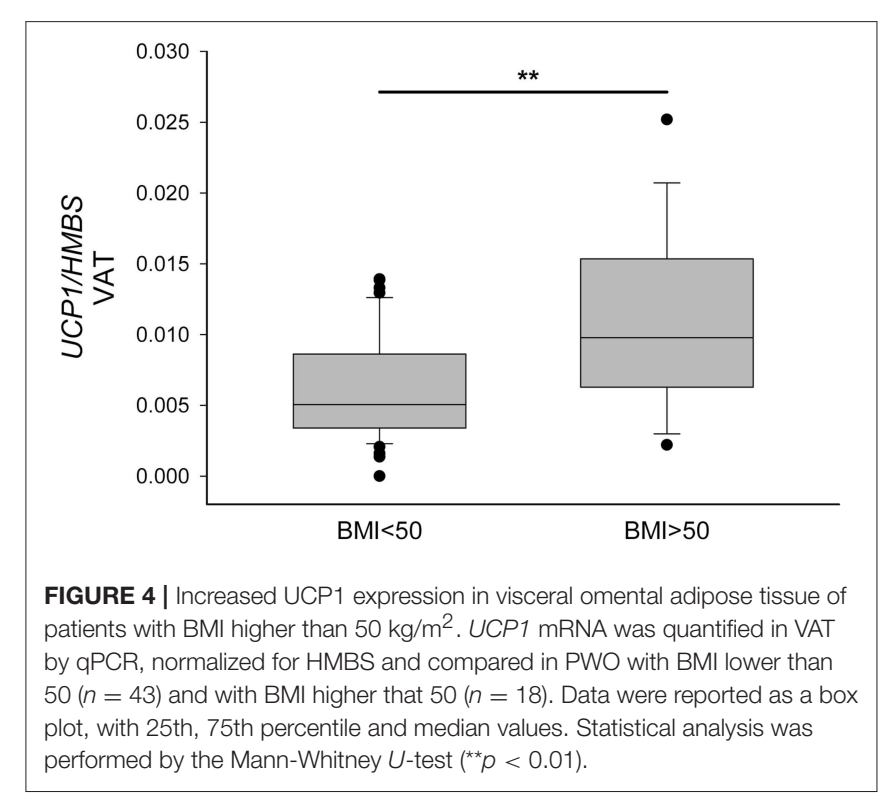

Two hundred and nine Caucasian PWO were enrolled at the Center for the Study and Integrated Treatment of Obesity, Padua University Hospital in the period 2013-2017, with a BMI $>30 \mathrm{~kg} / \mathrm{m}^{2}$. Patients underwent a multi-disciplinary evaluation according to a standard clinical protocol and a complete medical history was taken regarding eating, physical activity, smoking and drinking habits, drugs and medications, past and current medical conditions. PWO with a BMI $>35 \mathrm{~kg} / \mathrm{m}^{2}$ in the presence of co-morbidities or with a BMI $>40 \mathrm{~kg} / \mathrm{m}^{2}$ were candidates for bariatric surgery according to European criteria (50).

Specific exclusion criteria for this study were diagnosis of cancer in the previous 5 years, thyroid hormones imbalance, presence of infections or chronic inflammatory diseases, abuse of 
caffeine (more than three coffees/day), use of weight-loss drugs and other drugs that could interact with REE.

All subjects gave written informed consent in accordance with the Declaration of Helsinki. The protocol was approved by the "Padua Ethical Committee for Clinical Research" (2892P, 10/06/2013).

\section{Anthropometric Measurements}

All anthropometric measurements were taken with subjects wearing only light clothes without shoes. Height was measured to the nearest $0.01 \mathrm{~m}$ using a stadiometer. Body weight was determined to the nearest $0.1 \mathrm{~kg}$ using a calibrated balance beam scale. Waist circumference was assessed using a tape measure and BMI was calculated as weight $(\mathrm{kg})$ divided by heightsquared $\left(\mathrm{m}^{2}\right)$.

\section{Energy Expenditure}

REE was measured with indirect calorimetry in 209 PWO (described in Table 1) in fasting condition and after $15 \mathrm{~min}$ of rest in a comfortable and thermo-neutral environment. Rigorous attention to the standardization of measurement conditions was given, including ensuring that subjects avoided exercise, stressful situations or stimulants at defined intervals prior to the test $(51,52)$. A ventilated canopy calorimeter was used (Vmax Sensormedics, Milan, Italy). The system was calibrated before every measurement according to the instructions provided by the supplier. Oxygen uptake and carbon dioxide production were measured continuously and values were averaged at 1-min intervals. REE and respiratory quotient were calculated by using the Weir equation (53).

\section{Body Composition}

Body composition was analyzed with Body Impedance Assessment (BIA) by using a single frequency (300 $\mu \mathrm{A}$, $50 \mathrm{kHz}$ ) electrical impedance analyzer (Soft Tissue Analyzer, Akern, Pontassieve, Italy) in the same conditions as indirect calorimetry. Resistance (R), reactance (Xc) and the phase angle were registered and fat-free mass (FFM) and fat mass (FM) were derived using the software provided by the manufacturer (Bodygram software, Akern, Pontassieve, Italy).

\section{Biochemical Measurements}

For each patient we measured FPG, insulin, lipid profile [total cholesterol (TC), HDL- and Low Density Lipoprotein-cholesterol (LDL-cholesterol), triglycerides (TG)], hs-CRP, IL-6, Tumor Necrosis Factor- $\alpha$ (TNF- $\alpha$ ), and Leptin. All blood tests were performed after 8 -h fasting. Venous blood samples were collected in tubes coated with 68 IU lithium heparin, with EDTA 8\% or with acrylic gel/micronized silica in the morning (Becton Dickison, East Rutherford, NJ, USA). Samples were stored at $-20^{\circ} \mathrm{C}$ until analysis.

All biochemical blood analysis has been performed with standard diagnostic kit according to the WHO First International Reference Standard: glucose (Glucose HK Gen.3, Roche Diagnostic, USA), insulin, IL-6, TNF- $\alpha$ (IMMULITE 2000 Immunoassay, Siemens Healthcare GmbH, Germany), hs-CRP (CardioPhase High Sensitivity C-Reactive Protein,
TABLE 3 | Correlation and regression analyses of Resting Energy Expenditure (REE) in 35 patients with obesity.

\begin{tabular}{lcc}
\hline \multicolumn{2}{c}{ REE (kcal/day) } \\
\cline { 2 - 3 } & \multicolumn{2}{c}{ Simple linear coefficients $(\boldsymbol{r})$ Multiple regression coefficients $(\beta)$} \\
\hline Sex & -0.283 & 0.255 \\
WC & $0.406^{\star}$ & -0.0365 \\
FFM & $0.45^{\star \star}$ & $0.883^{\star \star \star}$ \\
FM & 0.181 & 0.321 \\
HOMA & $0.472^{\star \star}$ & $0.344^{\star}$ \\
HDL & -0.244 & $0.375^{\star}$ \\
IL-6 & 0.160 & $0.315^{\star}$ \\
UCP1-VAT & 0.1 & $-0.373^{\star}$ \\
\hline
\end{tabular}

WC, waist circumference; FFM, Free Fat Mass (kg); FM, Fat mass (kg); HOMA, Homeostasis model assessment-insulin resistance index; HDL, High Density Lipoprotein -cholesterol; IL-6, interleukin-6; UCP1-VAT, uncoupling protein 1 expression in visceral adipose tissue. Simple linear correlations were calculated by Pearson's correlation. We applied to this subgroup the same multiple regression model computed for the main population and introduced in the analysis UCP1-VAT as a possible biological implicating factor. FFM, HOMA, IL-6, HDL, and UCP1-VAT were independent factors correlated with $\operatorname{REE}(r=0.736$, AdjRsqr $=0.612) .{ }^{*} p<0.05 ;{ }^{* \star} p<0.01 ;{ }^{* \star \star} p<0.001$.

Siemens Healthcare), and Leptin (Leptin-RIA-CT, Mediagnost, Germany). Serum lipids were measured by spectrophotometer (Roche Diagnostic, USA). LDL cholesterol was calculated according to Friedewald (54). Non-HDL-cholesterol (CNHDL) has been obtained by the difference between TC and HDLcholesterol. A 3-h $75 \mathrm{~g}$ oral glucose tolerance test was performed monitoring blood glucose, insulin plasma levels at basal time and $30,90,120,150$, and $180 \mathrm{~min}$ after glucose load $(180 \mathrm{ml}$ of syrup with $82.5 \mathrm{~g}$ glucose monohydrate equal to $75 \mathrm{~g}$ of glucose) according to WHO standards (15).

HOMA was used to calculate the IR index by: [fasting serum insulin $(\mu \mathrm{U} / \mathrm{ml}) \times$ fasting plasma glucose $(\mathrm{mmol} / \mathrm{l})] / 22.5$, as previous described (55). In patients affected by T2DM, if insulin treated, fasting insulin was not measured and HOMA was not calculated.

\section{Human Adipose Tissue Samples}

During bariatric surgery paired SAT and VAT biopsies were collected in 62 PWO (described in Table 1), immediately frozen in liquid nitrogen and stored at $-80^{\circ} \mathrm{C}$ until RNA extraction. AT surrounding pheochromocytoma was collected during surgery for neoplasm removal.

\section{RNA Extraction and Reverse Transcription}

Total RNA was extracted using the RNeasy Lipid Tissue Mini Kit (QIAGEN GmbH, Hilden, Germany) following the supplier's instructions and quantified using NanoDrop (Thermo Fisher Scientific, Waltham, MA, USA).

One $\mu \mathrm{g}$ of RNA was treated with DNase Treatment \& Removal Reagents (Thermo Fisher Scientific) and reverse transcribed for $1 \mathrm{~h}$ at $37^{\circ} \mathrm{C}$ in a $50 \mu \mathrm{l}$ reaction containing $1 \mathrm{X}$ RT buffer, $150 \mathrm{ng}$ random hexamers, $0.5 \mathrm{mM}$ dNTPs, $20 \mathrm{U}$ RNAsin Ribonuclease Inhibitor, and $200 \mathrm{U}$ Moloney Murine Leukemia Virus reverse transcriptase (all from Promega Corp., Madison, WI, USA). 
TABLE 4 | qPCR conditions.

\begin{tabular}{|c|c|c|c|c|c|}
\hline Official symbol & Forward $\left(5^{\prime}-3^{\prime}\right)$ & Reverse $\left(5^{\prime}-3^{\prime}\right)$ & $\begin{array}{c}\text { Primer } \\
\text { (F/R nM) }\end{array}$ & Condition & $\begin{array}{c}\text { Amplicon } \\
\text { (bp) }\end{array}$ \\
\hline UCP1 & CTA CGA CAC GGT CCA GGA GT & GCC CAA TGA ATA CTG CCA CT & $300 / 300$ & $\begin{array}{c}95^{\circ} \mathrm{C} \times 15^{\prime \prime} \\
60^{\circ} \mathrm{C} \times 1^{\prime} \\
40 \text { cycles }\end{array}$ & 110 \\
\hline HMBS & GGC AAT GCG GCT GCA A & GGG TAC CCA CGC GAA TCA C & $300 / 300$ & $\begin{array}{c}95^{\circ} \mathrm{C} \times 15^{\prime \prime} \\
60^{\circ} \mathrm{C} \times 1^{\prime} \\
40 \text { cycles }\end{array}$ & 60 \\
\hline TFAM & AGC TCA GAA CCC AGA TGCA A & TाT ATA TAC CTG CCA CTC CGC C & $300 / 300$ & $\begin{array}{c}95^{\circ} \mathrm{C} \times 15^{\prime \prime} \\
60^{\circ} \mathrm{C} \times 1^{\prime} \\
40 \text { cycles }\end{array}$ & 127 \\
\hline TBX1 & ACG ACA ACG GCC ACA TTA TTC & TGA ATC GTG TCT CCT CGA ACA & $300 / 300$ & $\begin{array}{c}95^{\circ} \mathrm{C} \times 15^{\prime \prime} \\
60^{\circ} \mathrm{C} \times 1^{\prime} \\
40 \text { cycles }\end{array}$ & 141 \\
\hline
\end{tabular}

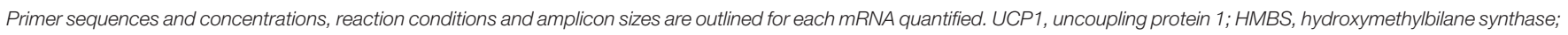
PPARGC1A, PPARG coactivator 1 alpha; TFAM, transcription factor A, mitochondrial; TBX1, T-box transcription factor 1; SLC27A1, solute carrier family 27 member 1.

\section{qPCR}

PCR was carried out with Platinum SYBR Green qPCR SuperMix-UDG (Thermo Fisher Scientific) on DNA Engine OpticonTM2 Continuous Fluorescence Detection System (MJ Research, MA, USA). Reaction conditions and primer sequences were reported in Table 4. Each sample (5 ng of cDNA) was assayed in duplicate and quantified using a standard curve method. Results were normalized to HMBS mRNA content and reported as arbitrary unit ratio (target/housekeeping). The melting curve analysis and a positive control (UCP1: VAT surrounding pheochromocytoma, PPARGC1A and TFAM: liver, TBX1: muscle, SLC27A1: white adipose tissue) were always included to check PCR specificity.

\section{Statistical Analysis}

Statistical analyses were performed using the Systat Software SigmaPlot v.13. Data are presented as mean values \pm standard deviations or as median values (25th-75th percentile). All variables were tested by normal Test (Shapiro-Wilk test) and Equal Variance Test (Brown-Forsythe). Pearson's correlation coefficient $(r)$ and the relative $p$-values were calculated to analyze simple linear correlations between two variables. A multiple regression model was computed with REE as the dependent variable and the variables found to be simple correlated with REE as independent variables. This model was used to analyze a subgroup of 35 PWO in which we quantified also UCP1 mRNA in VAT biopsies (UCP1-VAT) and entered this new variable as an independent variable. Differences between the population of 209 patients vs. the subgroup of 35 patients were analyzed by Mann-Whitney $U$-test for independent samples in non-normally distributed variables, independent $t$-test in normally distributed variables and Fisher's exact test in categorical variables. Differences between UCP1 expression in VAT and SAT were analyzed by the Mann-Whitney $U$-test. In all analyses, the $p$-values were two-sided and a $p$-value lower than 0.05 was considered statistically significant.

\section{DATA AVAILABILITY}

The datasets generated for this study are available on request to the corresponding author.

\section{ETHICS STATEMENT}

All subjects gave written informed consent in accordance with the Declaration of Helsinki. The protocol was approved by the Padua Ethical Committee for Clinical Research (2892P, 10/06/2013).

\section{AUTHOR CONTRIBUTIONS}

SB and FF conceptualized the study, the methodology, and prepared the original draft. SB, FF, LB, AB, and MS participated in the data curation and the statistical analyses. CC, RF, RS, CD, LP, and MF provided the clinical data and samples. RV, GM, and LB supervised the study, reviewed, and edited the manuscript.

\section{FUNDING}

This research was funded by PRIN from the Italian MIUR, grant number 2015JSWLTN_002, to LB; grant number 2017L8Z2EM, to RV. 


\section{REFERENCES}

1. Müller MJ, Geisler C, Hübers M, Pourhassan M, Braun W, BosyWestphal A. Normalizing resting energy expenditure across the life course in humans: challenges and hopes. Eur J Clin Nutr. (2018) 72:62837. doi: 10.1038/s41430-018-0151-9

2. Bosy-Westphal A, Wolf A, Bührens F, Hitze B, Czech N, Mönig H, et al. Familial influences and obesity-associated metabolic risk factors contribute to the variation in resting energy expenditure: the Kiel Obesity Prevention Study. Am J Clin Nutr. (2008) 87:1695-701. doi: 10.1093/ajcn/87.6.1695

3. Drabsch T, Holzapfel C, Stecher L, Petzold J, Skurk T, Hauner H. Associations between C-reactive protein, insulin sensitivity, and resting metabolic rate in adults: a mediator analysis. Front Endocrinol. (2018) 9:556. doi: 10.3389/fendo.2018.00556

4. Bitz C, Toubro S, Larsen TM, Harder H, Rennie KL, Jebb SA, et al. Increased 24-h energy expenditure in type 2 diabetes. Diabetes Care. (2004) 27:241621. doi: $10.2337 /$ diacare.27.10.2416

5. Alawad AO, Merghani TH, Ballal MA. Resting metabolic rate in obese diabetic and obese non-diabetic subjects and its relation to glycaemic control. BMC Res Notes. (2013) 6:382. doi: 10.1186/1756-0500-6-382

6. Fontvieille AM, Lillioja S, Ferraro RT, Schulz LO, Rising R, Ravussin E. Twenty-four-hour energy expenditure in Pima Indians with type 2 (noninsulin-dependent) diabetes mellitus. Diabetologia. (1992) 35:753-9.

7. Lowell BB, Spiegelman BM. Towards a molecular understanding of adaptive thermogenesis. Nature. (2000) 404:652-60. doi: 10.1038/35007527

8. Ouellet V, Labbé SM, Blondin DP, Phoenix S, Guérin B, Haman F, et al. Brown adipose tissue oxidative metabolism contributes to energy expenditure during acute cold exposure in humans. J Clin Invest. (2012) 122:54552. doi: 10.1172/JCI60433

9. Chondronikola M, Volpi E, Børsheim E, Porter C, Annamalai P, Enerbäck S, et al. Brown adipose tissue improves whole-body glucose homeostasis and insulin sensitivity in humans. Diabetes. (2014) 63:4089-99. doi: 10.2337/db14-0746

10. Kajimura S, Spiegelman BM, Seale P. Brown and beige fat: physiological roles beyond heat generation. Cell Metab. (2015) 22:546-59. doi: 10.1016/j.cmet.2015.09.007

11. Després J-P, Lemieux I. Abdominal obesity and metabolic syndrome. Nature. (2006) 444:881-7. doi: 10.1038/nature05488

12. Seale P, Conroe HM, Estall J, Kajimura S, Frontini A, Ishibashi J, et al. Prdm16 determines the thermogenic program of subcutaneous white adipose tissue in mice. J Clin Invest. (2011) 121:96-105. doi: 10.1172/JCI44271

13. Zuriaga MA, Fuster JJ, Gokce N, Walsh K. Humans and mice display opposing patterns of "browning" gene expression in visceral and subcutaneous white adipose tissue depots. Front Cardiovasc Med. (2017) 4:27. doi: 10.3389/fcvm.2017.00027

14. Rockstroh D, Landgraf K, Wagner IV, Gesing J, Tauscher R, Lakowa N, et al. Direct evidence of brown adipocytes in different fat depots in children. PLoS ONE. (2015) 10:e0117841. doi: 10.1371/journal.pone.0117841

15. American Diabetes Association. Diagnosis and classification of diabetes mellitus. Diabetes Care. (2014) 37 (Suppl. 1):S81-90. doi: 10.2337/dc14-S081

16. Frontini A, Vitali A, Perugini J, Murano I, Romiti C, Ricquier D, et al. White-to-brown transdifferentiation of omental adipocytes in patients affected by pheochromocytoma. Biochim Biophys Acta. (2013) 1831:9509. doi: 10.1016/j.bbalip.2013.02.005

17. Weyer C, Bogardus C, Pratley RE. Metabolic factors contributing to increased resting metabolic rate and decreased insulin-induced thermogenesis during the development of type 2 diabetes. Diabetes. (1999) 48:160714. doi: $10.2337 /$ diabetes.48.8.1607

18. Swinburn BA, Nyomba BL, Saad MF, Zurlo F, Raz I, Knowler WC, et al. Insulin resistance associated with lower rates of weight gain in Pima Indians. J Clin Invest. (1991) 88:168-73. doi: 10.1172/JCI115274

19. Piaggi P, Thearle MS, Bogardus C, Krakoff J. Fasting hyperglycemia predicts lower rates of weight gain by increased energy expenditure and fat oxidation rate. J Clin Endocrinol Metab. (2015) 100:1078-87. doi: 10.1210/jc.2014-3582

20. Franssila-Kallunki A, Groop L. Factors associated with basal metabolic rate in patients with type 2 (non-insulin-dependent) diabetes mellitus. Diabetologia. (1992) 35:962-6.
21. Bettini S, Bordigato E, Fabris R, Serra R, Dal Pra' C, Belligoli A, et al. Modifications of resting energy expenditure after sleeve gastrectomy. Obes Surg. (2018) 28:2481-6. doi: 10.1007/s11695-018-3190-3

22. Sampath Kumar A, Arun Maiya G, Shastry BA, Vaishali K, Maiya S, Umakanth S. Correlation between basal metabolic rate, visceral fat and insulin resistance among type 2 diabetes mellitus with peripheral neuropathy. Diabetes Metab Syndr. (2019) 13:344-8. doi: 10.1016/j.dsx.2018.10.005

23. Pouliot MC, Després JP, Lemieux S, Moorjani S, Bouchard C, Tremblay A, et al. Waist circumference and abdominal sagittal diameter: best simple anthropometric indexes of abdominal visceral adipose tissue accumulation and related cardiovascular risk in men and women. Am J Cardiol. (1994) 73:460-8. doi: 10.1016/0002-9149(94)90676-9

24. Busetto L, Perini P, Giantin V, Valente P, Segato G, Belluco C, et al. Relationship between energy expenditure and visceral fat accumulation in obese women submitted to adjustable silicone gastric banding (ASGB). Int J Obes Relat Metab Disord. (1995) 19:227-33.

25. Alberti KGMM, Zimmet P, Shaw J, IDF Epidemiology Task Force Consensus Group. The metabolic syndrome-a new worldwide definition. Lancet. (2005) 366:1059-62. doi: 10.1016/S0140-6736(05)67402-8

26. Hickmann CE, Roeseler J, Castanares-Zapatero D, Herrera EI, Mongodin A, Laterre P-F. Energy expenditure in the critically ill performing early physical therapy. Intensive Care Med. (2014) 40:548-55. doi: 10.1007/s00134-014-3218-7

27. Utaka S, Avesani CM, Draibe SA, Kamimura MA, Andreoni S, Cuppari L. Inflammation is associated with increased energy expenditure in patients with chronic kidney disease. Am J Clin Nutr. (2005) 82:8015. doi: 10.1093/ajcn/82.4.801

28. Pradhan AD, Manson JE, Rifai N, Buring JE, Ridker PM. C-reactive protein, interleukin 6, and risk of developing type 2 diabetes mellitus. JAMA. (2001) 286:327-34. doi: 10.1001/jama.286.3.327

29. Mauer J, Denson JL, Brüning JC. Versatile functions for IL-6 in metabolism and cancer. Trends Immunol. (2015) 36:92-101. doi: 10.1016/j.it.2014.12.008

30. Stanford KI, Middelbeek RJW, Townsend KL, An D, Nygaard EB, Hitchcox $\mathrm{KM}$, et al. Brown adipose tissue regulates glucose homeostasis and insulin sensitivity. J Clin Invest. (2013) 123:215-23. doi: 10.1172/JCI62308

31. Kristóf E, Klusóczki Á, Veress R, Shaw A, Combi ZS, Varga K, et al. Interleukin-6 released from differentiating human beige adipocytes improves browning. Exp Cell Res. (2019) 377:47-55. doi: 10.1016/j.yexcr.2019. 02.015

32. Jorge ASB, Jorge GCB, Paraíso AF, Franco RMP, Vieira LJT, Hilzenderger AM, et al. Brown and white adipose tissue expression of IL6, UCP1 and SIRT1 are associated with alterations in clinical, metabolic and anthropometric parameters in obese humans. Exp Clin Endocrinol Diabetes. (2017) 125:16370. doi: $10.1055 / \mathrm{s}-0042-119525$

33. Alcalá M, Calderon-Dominguez M, Bustos E, Ramos P, Casals N, Serra $\mathrm{D}$, et al. Increased inflammation, oxidative stress and mitochondrial respiration in brown adipose tissue from obese mice. Sci Rep. (2017) 7:16082. doi: 10.1038/s41598-017-16463-6

34. García-Ruiz E, Reynés B, Díaz-Rúa R, Ceresi E, Oliver P, Palou A. The intake of high-fat diets induces the acquisition of brown adipocyte gene expression features in white adipose tissue. Int J Obes. (2015) 39:161929. doi: 10.1038/ijo.2015.112

35. Patsouris D, Qi P, Abdullahi A, Stanojcic M, Chen P, Parousis A, et al. Burn induces browning of the subcutaneous white adipose tissue in mice and humans. Cell Rep. (2015) 13:1538-44. doi: 10.1016/j.celrep.2015.10.028

36. Petruzzelli M, Schweiger M, Schreiber R, Campos-Olivas R, Tsoli M, Allen J, et al. A switch from white to brown fat increases energy expenditure in cancer-associated cachexia. Cell Metab. (2014) 20:43347. doi: 10.1016/j.cmet.2014.06.011

37. Saito M, Okamatsu-Ogura Y, Matsushita M, Watanabe K, Yoneshiro T, NioKobayashi J, et al. High incidence of metabolically active brown adipose tissue in healthy adult humans: effects of cold exposure and adiposity. Diabetes. (2009) 58:1526-31. doi: 10.2337/db09-0530

38. Brendle C, Werner MK, Schmadl M, la Fougère C, Nikolaou K, Stefan N, et al. Correlation of brown adipose tissue with other body fat compartments and patient characteristics: a retrospective analysis in a large patient cohort using PET/CT. Acad Radiol. (2018) 25:102-10. doi: 10.1016/j.acra.2017.09.007 
39. Cypess AM, Lehman S, Williams G, Tal I, Rodman D, Goldfine AB, et al. Identification and importance of brown adipose tissue in adult humans. $N$ Engl J Med. (2009) 360:1509-17. doi: 10.1056/NEJMoa0810780

40. Wu J, Boström P, Sparks LM, Ye L, Choi JH, Giang AH, et al. Beige adipocytes are a distinct type of thermogenic fat cell in mouse and human. Cell. (2012) 150:366-76. doi: 10.1016/j.cell.2012.05.016

41. Kraunsøe R, Boushel R, Hansen CN, Schjerling P, Qvortrup K, Støckel $\mathrm{M}$, et al. Mitochondrial respiration in subcutaneous and visceral adipose tissue from patients with morbid obesity. J Physiol. 2010588 (Pt 12):202332. doi: 10.1113/jphysiol.2009.184754

42. Kelley DE, He J, Menshikova EV, Ritov VB. Dysfunction of mitochondria in human skeletal muscle in type 2 diabetes. Diabetes. (2002) 51:294450. doi: 10.2337/diabetes.51.10.2944

43. Charlton MR, Nair KS. Role of hyperglucagonemia in catabolism associated with type 1 diabetes: effects on leucine metabolism and the resting metabolic rate. Diabetes. (1998) 47:1748-56. doi: 10.2337/diabetes.47.11.1748

44. Lam TKT, Schwartz GJ, Rossetti L. Hypothalamic sensing of fatty acids. Nat Neurosci. (2005) 8:579-84. doi: 10.1038/nn1456

45. Kazak L, Chouchani ET, Jedrychowski MP, Erickson BK, Shinoda $\mathrm{K}$, Cohen $\mathrm{P}$, et al. A creatine-driven substrate cycle enhances energy expenditure and thermogenesis in beige fat. Cell. (2015) 163:643-55. doi: 10.1016/j.cell.2015.09.035

46. Ikeda K, Kang Q, Yoneshiro T, Camporez JP, Maki H, Homma M, et al. UCP1-independent signaling involving SERCA2b-mediated calcium cycling regulates beige fat thermogenesis and systemic glucose homeostasis. Nat Med. (2017) 23:1454-65. doi: 10.1038/nm.4429

47. Chen Y, Zeng X, Huang X, Serag S, Woolf CJ, Spiegelman BM. Crosstalk between KCNK3-Mediated Ion Current and Adrenergic Signaling Regulates Adipose Thermogenesis and Obesity. Cell. (2017) 171:836848.e13. doi: 10.1016/j.cell.2017.09.015

48. Ukropec J, Anunciado RP, Ravussin Y, Hulver MW, Kozak LP. UCP1independent thermogenesis in white adipose tissue of cold-acclimated Ucp1-/- mice. J Biol Chem. (2006) 281:31894-908. doi: 10.1074/jbc.M6061 14200
49. Granneman JG, Burnazi M, Zhu Z, Schwamb LA. White adipose tissue contributes to UCP1-independent thermogenesis. Am J Physiol Endocrinol Metab. (2003) 285:E1230-6. doi: 10.1152/ajpendo.00197.2003

50. Yumuk V, Tsigos C, Fried M, Schindler K, Busetto L, Micic D, et al. Obesity management task force of the european association for the study of obesity. european guidelines for obesity management in adults. Obes Facts. (2015) 8:402-24. doi: 10.1159/000442721

51. Ferrannini E. The theoretical bases of indirect calorimetry: a review. Metab Clin Exp. (1988) 37:287-301.

52. Branson RD, Johannigman JA. The measurement of energy expenditure. Nutr Clin Pract. (2004) 19:622-36. doi: 10.1177/0115426504019006622

53. Weir JBDB. New methods for calculating metabolic rate with special reference to protein metabolism. J Physiol. (1949) 109:1-9. doi: 10.1113/jphysiol.1949.sp004363

54. Friedewald WT, Levy RI, Fredrickson DS. Estimation of the concentration of low-density lipoprotein cholesterol in plasma, without use of the preparative ultracentrifuge. Clin Chem. (1972) 18:499-502.

55. Matthews DR, Hosker JP, Rudenski AS, Naylor BA, Treacher DF, Turner RC. Homeostasis model assessment: insulin resistance and beta-cell function from fasting plasma glucose and insulin concentrations in man. Diabetologia. (1985) 28:412-9.

Conflict of Interest Statement: The authors declare that the research was conducted in the absence of any commercial or financial relationships that could be construed as a potential conflict of interest.

Copyright (c) 2019 Bettini, Favaretto, Compagnin, Belligoli, Sanna, Fabris, Serra, Dal Prà, Prevedello, Foletto, Vettor, Milan and Busetto. This is an open-access article distributed under the terms of the Creative Commons Attribution License (CC BY). The use, distribution or reproduction in other forums is permitted, provided the original author(s) and the copyright owner(s) are credited and that the original publication in this journal is cited, in accordance with accepted academic practice. No use, distribution or reproduction is permitted which does not comply with these terms. 